\title{
New Yb:LYSB bifunctional crystal for efficient near-infrared laser emission and self-frequency doubling conversion
}

\author{
Alin Broasca ${ }^{1,2}$, Madalin Greculeasa ${ }^{1,2}$, Flavius Voicu ${ }^{1}$, Stefania Hau ${ }^{1}$, Gabriela Croitoru ${ }^{1}$, \\ Cristina Gheorghe ${ }^{1}$, Nicolaie Pavel ${ }^{1}$, Lucian Gheorghe ${ }^{1}$ \\ 1. National Institute for Laser, Plasma and Radiation Physics, Laboratory of Solid-State Quantum Electronics, Magurele 077125, Romania \\ 2. Doctoral School of Physics, University of Bucharest, Faculty of Physics, Magurele 077125, Romania
}

Nowadays, solid-state lasers with emission in the visible (VIS) spectral range are used in a large variety of applications, from manufacturing and materials processing technologies to medicine, optical communication or military applications. A widely used method for the obtaining of efficient laser sources in VIS range is based on frequency conversion of solid-state near-infrared laser emission by nonlinear optical (NLO) processes, such as second harmonic generation (SHG) or self-frequency doubling (SFD).

In this work, we report on the development of Yb:LYSB as new bifunctional laser and NLO crystal. Based on our experience on growing undoped $\mathrm{La}_{\mathrm{x}} \mathrm{Y}_{\mathrm{y}} \mathrm{Sc}_{4-\mathrm{x}-\mathrm{y}}\left(\mathrm{BO}_{3}\right)_{4}-\mathrm{LYSB}$ - type crystals [1], a special heating assembly that allows an effective control of both $\mathrm{B}_{2} \mathrm{O}_{3}$ evaporation and thermal gradients over the crucible and in the melt, was used for the growth of the incongruent melting Yb:LYSB crystal by the Czochralski method (Fig. 1). The XRD analysis revealed the existence of the single trigonal phase, space group $R 32$; the unit cell parameters were determined to be $\mathrm{a}=9.8114(4) \AA$ and $\mathrm{c}=7.9808(9) \AA$. The chemical composition of the crystal was found to be $\mathrm{La}_{0.78} \mathrm{Y}_{0.32} \mathrm{Yb}_{0.04} \mathrm{Sc}_{2.86}\left(\mathrm{BO}_{3}\right)_{4}$. The segregation coefficient of $\mathrm{Yb}^{3+}$ ions in the LYSB crystal matrix was determined to be $\mathrm{k}_{\mathrm{eff}}=0.8$, corresponding to an $\mathrm{Yb}^{3+}$-doping concentration of 4 at.\% in the grown crystal. The absorption cross-section, $\sigma_{\mathrm{a}}$ at $980 \mathrm{~nm}$ for $\sigma$-polarization is about $0.6 \times 10^{-20} \mathrm{~cm}^{2}$ with a bandwidth (FWHM definition) of $33 \mathrm{~nm}$, being $\sim 1.65$ times larger than that of Yb:YAB crystal [2]. In the case of $\pi$-polarization, the highest $\sigma_{\mathrm{a}}$ was determined to be $0.37 \times 10^{-20} \mathrm{~cm}^{2}$ at $904 \mathrm{~nm}$, being close to the value reported for Yb:LGSB crystal [3]. Furthermore, spectroscopic studies revealed an intrinsic disorder due to the presence of $\mathrm{Yb}^{3+}$ ions in the trigonal prismatic sites occupied by $\mathrm{La}^{3+}$ and $\mathrm{Y}^{3+}$ ions and also in the octahedral sites occupied by $\mathrm{Sc}^{3+}$ ions.

Efficient laser emission at the fundamental wavelength $\lambda_{\mathrm{em}}=1028 \mathrm{~nm}$ was obtained with a $\boldsymbol{c}$-cut 4 -at.\% $\mathrm{Yb}:$ LYSB uncoated medium, having a thickness of $3.5 \mathrm{~mm}$. The pump was done at $971.5 \mathrm{~nm}$ with a fibercoupled $(100-\mu \mathrm{m}$ diameter, $\mathrm{NA}=0.22)$ diode laser. A short (8-mm length) linear plane-plane resonator was used in the experiments. With an out-coupling mirror having transmission $\mathrm{T}=0.01$, the $\mathrm{Yb}$ :LYSB medium yielded laser pulses with maximum energy $\mathrm{E}_{\mathrm{p}}=1.5 \mathrm{~mJ}$ at $4.25 \mathrm{~mJ}$ energy of the absorbed pump pulse, $\mathrm{E}_{\mathrm{abs}}$; thus, overall optical-to-optical efficiency, with respect to $E_{a b s}$, was $\eta_{\mathrm{oa}}=0.35$. The slope efficiency was as high as $\eta_{\mathrm{sa}}=0.57$ (Fig. 2), proving the good optical quality of Yb:LYSB grown crystal.

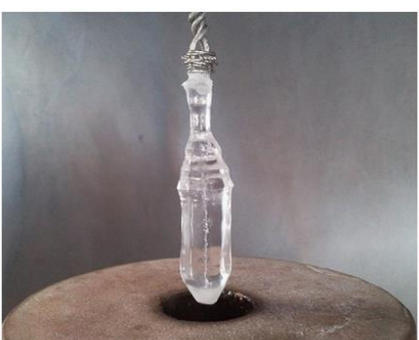

Fig. 1 Yb:LYSB as-grown crystal

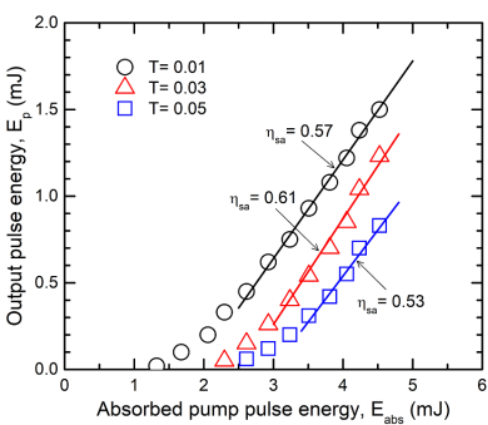

Fig. 2 Laser pulse energy, $E_{p}$ vs. absorbed energy of the pump pulse, $\mathrm{E}_{\mathrm{abs}} \mathrm{T}$ is the out-coupling mirror transmission.

The NLO properties of Yb:LYSB crystal were determined from the refractive indices measurements. The effective nonlinear coefficient, $d_{\text {eff }}$ for type I SHG of $\lambda_{\mathrm{em}}=1028 \mathrm{~nm}$ was determined to be $1.08 \mathrm{pm} / \mathrm{V}$, confirming the good NLO properties of huntite-type Yb:LYSB crystal. Further experiments to determine the full potential of $\mathrm{Yb}$ :LYSB crystal as bifunctional laser and NLO crystal in SFD configuration are now in progress.

Financing: Projects 119/2017, PN-III-P4-ID-PCE-2016-0853 and NUCLEU 16N/2019, Ministry of Research and Innovation, Romania.

\section{References}

[1] L. Gheorghe, M. Greculeasa, A. Broasca, F. Voicu, G. Stanciu, K. N. Belikov, E. Yu. Bryleva, O. Gaiduk, "Incongruent Melting $\mathrm{La}_{\mathrm{x}} \mathrm{Y}_{\mathrm{y}} \mathrm{Sc}_{4-\mathrm{x}-\mathrm{y}}\left(\mathrm{BO}_{3}\right)_{4}$ : LYSB Nonlinear Optical Crystal Grown by the Czochralski Method," ACS Appl. Mater. Interfaces 11(23), 2098720994 (2019).

[2] P. Wang, J.M. Dawes, P. Dekker, D.S. Knowles, J.A. Piper, B. Lu, "Growth and evaluation of ytterbium-doped yttrium aluminum borate as a potential self-doubling laser crystal," J. Opt. Soc. Am. B 16(1), 63-69 (1999).

[3] F. Khaled, P. Loiseau, F. Voicu, A. Achim, S. Hau, C. Gheorghe, G. Croitoru, N. Pavel, L. Gheorghe, G. Aka, "Spectroscopic properties and laser performances of Yb:LGSB nonlinear optical crystal," J. Alloy. Compd. 688(Part A), 510-517 (2016). 